\title{
MODELING METHODOLOGY FOR PHYSICS TEACHERS
}

\author{
David Hestenes \\ Department of Physics and Astronomy \\ Arizona State University \\ Tempe, Arizona 85287-1504
}

\begin{abstract}
Scientific practice involves the construction, validation and application of scientific models, so science instruction should be designed to engage students in making and using models. Scientific models are coherent units of structured knowledge. They are used to organize factual information into coherent wholes, often by the coordinated use of general laws or principles. Therefore, the structure of scientific knowledge can be made more explicit for students by organizing course content around a small number of basic models. The ability of students to make and use models depends on the representational tools at their command. Students learn transferable modeling skills by applying given models to a variety of situations to describe, explain, or predict physical events or to design experiments. These ideas have been incorporated into a methodology for physics teaching and a course for training teachers.
\end{abstract}

\section{Introduction}

This paper describes theoretical underpinnings for a two-semester course in the methodology of physics teaching designed especially for in-service and preservice high school teachers but valuable for regular physics majors as well. Currently the course is being disseminated nationally in a series of summer "Modeling Workshops" supported by the National Science Foundation. The first two-summer series, held at university sites in Tempe and Chicago, was completed in 1996. A second series begins in summer 1997 at three new sites, and a third begins in 1998 at three more sites. Information about the workshops can be obtained by email from <jane.jackson@asu.edu> or by visiting our web site at http://modeling.la.asu.edu/modeling.html. Incorporation of the courses into the university physics curriculum is discussed by Hestenes and Jackson (7).

The Modeling Workshops have evolved over more than a decade from educational research and the experience of exceptional physics teachers. The story has been told in (16). This paper updates and elaborates the methodology, which is still evolving. Unfortunately, a full understanding of the "Modeling Method" cultivated in the Workshops cannot be acquired from a written description. Participation in a Workshop is essential. This paper gives an overview of the pedagogical framework with selective emphasis on a few important issues. For a more complete account the references should be consulted.

\section{Course Objectives and Scope}

The main purpose of the Modeling Workshops is to empower teachers with a robust teaching methodology. This includes the cultivation of teacher abilities to critically analyze any given curriculum materials and organize valuable 
parts into effective instructional units which make the underlying models explicit- tasks which require a strong pedagogical framework. These abilities are needed to take advantage of accelerating changes in curriculum materials, driven on the one hand by advances in educational research and by new computer technology and software on the other.

Workshops I and II have different designs to promote complementary pedagogical objectives. The main objective of Workshop I (on mechanics) is to acquaint teachers with all aspects of the modeling method and develop skill in implementing it. To that end, they are provided with a fairly complete set of written curriculum materials to support instruction organized into coherent modeling cycles (as described in (16)). The physical materials and experiments in the curriculum are simple and quite standard, already available in any reasonably equipped physics classroom. In due time the teachers come to understand that the modeling method can be applied as well to more unusual, complex, and exciting subjects. Since "teachers teach as they have been taught," workshops include extensive practice in implementing the curriculum as intended for their classes. They circulate through roles of student and instructor as they practice techniques of guided inquiry and collaborative learning.

The design of the Second Workshop (on light, electricity, heat, ...) is more fluid and open-ended. The main objective is to give the teachers intensive experience in using what they have learned about the modeling method to construct and evaluate coherent instructional units of their own design. They must first learn how to construct such units from standard curriculum materials, already available or easily procured for their classes. For each of the major topics, small groups of teachers study available curriculum materials. They are guided to identify the underlying models and develop coherent instructional units which engage students in using the models to structure their own understanding. The units developed by the various groups are presented to the entire class for critique, evaluation and discussion of how to organize the units into a complete curriculum.

Instructional materials developed and disseminated in the Workshops are secondary to the greater goal of developing a flexible teaching methodology receptive to new materials, especially in the nascent domain of physics education software. This point deserves emphasis, because there is an unfortunate tendency in some quarters to equate educational reform with the creation and distribution of new materials. The Modeling Workshops aim to promote a community of reformminded teachers who will continue upgrading their own curriculum materials and classroom practice - who are motivated by the vision of a dynamic teacher empowered with special skills, rather than by the static "Here's a set of materials, use 'em!"

\section{Where Physics Teaching Fails: Evaluating Instruction}

Physicists appreciate the need for instruments which produce accurate and reproducible measurements. Reif (1996) has called for the same in physics 
teaching. He contends that we need operationally specified performance standards for comparing the effectiveness of different teaching methods. This calls for development and calibration of a battery of instruments for evaluating instruction in a variety of ways. The Force Concept Inventory (FCI) is one such instrument with proven value (8).

The FCI was developed to assess the effectiveness of mechanics courses in meeting a minimal performance standard: to teach students to reliably discriminate between the applicability of scientific concepts and naive alternatives in common physical situations. The FCI systematically probes student abilities to make such discriminations with respect to six fundamental aspects of the Newtonian force concept.

Including the recent survey by Hake (3), we now have FCI data on more than 12,000 students and 100 physics courses in high schools, colleges and universities through the United States. This large data base presents a highly consistent picture, showing that the FCI provides statistically reliable and discriminating measures of minimal performance in mechanics. The results strongly support the following general conclusions:

- Before physics instruction, students hold naive beliefs about mechanics which are incompatible with Newtonian concepts in most respects.

- Such beliefs are a major determinant of student performance in introductory physics.

- Traditional (lecture-demonstration) physics instruction induces only a small change in the beliefs. This result is largely independent of the instructor's knowledge, experience and teaching style.

- Much greater changes in student beliefs can be induced with instructional methods derived from educational research.

These conclusions can be quantified. From pre/post course FCI scores of 14 traditional courses, Hake (3) found a mean normalized gain of $22 \%$, with a largest gain of $32 \%$. In contrast, for 41 courses using non-traditional teaching methods, he found a mean gain of 52\%, with a largest gain of $69 \%$. The two means differ by several standard deviations - a highly significant result. This shows that traditional instruction fails badly in meeting a minimal performance standard for mechanics. Moreover, the failure cannot be attributed to inadequacies of the students, for the data show that alternative methods of instruction can do much better. A major problem in physics education research for more than a decade has been to identify essential conditions for learning Newtonian physics and therefrom devise more effective teaching methods.

To understand the significance of the low FCI scores for traditional instruction, consider the results in Box 1 which have been extracted from FCI data. These results (which can be supported by a much more extensive analysis) show that most students complete introductory physics, even at the university level, with a seriously deficient understanding of the Newtonian force concept. The consequences of this fact are devastating. Unaware that their own ideas 
about force differ drastically from those of the teacher, most students systematically misunderstand what they hear and read in traditional introductory physics. Consequently, they cannot understand why they fail at problem solving, and they are forced to resort to rote methods for learning meaningless formulas and procedures. The result is frustration, humiliation and student turnoff!

Fortunately, educational research has shown us how to do better. Indeed, we have documented that several teachers using the modeling method have achieved FCI gains within and even surpassing the highest performance range in Hake's data set. Details will be published elsewhere.

\section{Understanding Physics: A Question of Structure}

One reason for the failure of traditional instruction is that it overlooks the crucial influence of students' personal beliefs on what they learn. In the traditional setting naive student beliefs about physics are labeled as misconceptions and are likely to be summarily dismissed as unworthy of consideration. However, the FCI data show that students are not easily induced to discard their misconceptions in favor of Newtonian concepts. Indeed, physics teachers and educational researchers who are aware of this problem have expended considerable effort in designing and testing teaching methods to deal with specific misconceptions. Although their outcomes have been decidedly better than the traditional ones, success has been limited and the methods can be criticized as excessively time and labor intensive. Many have concluded that student beliefs are so "deepseated" that heavy instructional costs to unseat them are unavoidable. However, documented success with the modeling method (16) suggest that an indirect treatment of misconceptions is likely to be most efficient and effective. In any case, an optimal solution of the problem will surely require an understanding of the cognitive factors involved.

Our analysis of the cognitive factors begins with the observation that the student "misconceptions" in Box 1 are framed in terms of metaphors. Cognitive scientists (10) have identified metaphors as a fundamental tool of human thought, which we use so frequently and automatically that we seldom notice the metaphors unless they are called to our attention. Metaphors are used to structure our experience and thereby make it meaningful. In particular, metaphors help us make sense of new experience (target domain) by mapping it onto structure of familiar experience (source domain). Johnson (9) argues that our bodily experience, structurally represented by mental image schemas, is the source for our strongest metaphors. For example, we use prepositions to construct a rich system of spatial metaphors, like "more is up," which are grounded in such schemas. Johnson argues further that the metaphorical use of image schemas is pervasive in our understanding of abstract ideas, including mathematics. Even the idea of "deduction" derives from the spatial concept of "following a path."

This analysis suggests that the source domain for the metaphors in Box 1 is indeed deep-seated, but that the mapping to the target domain is more compliant and may adjust fairly quickly to make sense of new experience under 


\begin{tabular}{|llcl|}
\hline Newtonian Concepts & vs. & Naive Beliefs & Results \\
\hline First Law & vs. & $\begin{array}{c}\text { "Motion requires force" } \\
\text { (Impetus Principle) }\end{array}$ & $(\sim 60 \%)$ \\
Third Law & vs. & $\begin{array}{c}\text { "Force is action" } \\
\text { (No Passive forces) } \\
\text { "Force is war" } \\
\text { (Dominance Principle) }\end{array}$ & $(\sim 40 \%)$ \\
\hline
\end{tabular}

BOX 1. Major beliefs about forces. The percentages are rough estimates of typical post-instruction results for traditional instruction in University (calculusbased) Physics at a large state university. They indicate the fraction of students who fail to consistently discriminate an appropriate use of Newton's Laws from naive alternatives.

appropriate conditions. In other works, misconceptions about mechanics are just cases of misplaced metaphors which will be corrected automatically in response to appropriate experience. The problem of instruction is therefore to arrange the conditions for such experience. This perspective provides a rationale for one of the most systematic methods for treating misconceptions. Clement and Camp (2) engage students in constructing a series of "bridging analogies" which directs their attention to commonalties in a variety of physical situations.

It has been suggested that student misconceptions about mechanics are fundamentally different than misconceptions about electricity and magnetism, because students have so much more experience with moving objects. Our present perspective on metaphors suggests otherwise. Students automatically use metaphors to structure experience in any scientific domain. The case of mechanics shows that when they do this casually the metaphors are likely to be misplaced even in domains where they have extensive experience. A major objective of teaching should therefore be to help students "straighten out" their metaphors. This readjustment of mappings onto their personal image schemas can be regarded as developing physical intuition.

Physics teachers know the importance of "physical intuition," but they should be aware that their intuitions (metaphorical mappings) are not the same as their students'. Therefore, when they express their intuition about force by describing it as "a push or a pull," they are likely to evoke in students a metaphorical association of force with human action, one of the major misconceptions in Box 1.

As explained in the following sections, the modeling method approaches the problem of restructuring students' intuitions by engaging them in explicit construction and manipulation of externally structured representations. In the case of mechanics (6), we have found it advisable to engage students in explicit comparisons of the three major misconceptions in Box 1 with their Newtonian alternatives. When these three are adequately treated, many other misconceptions about mechanics fall away with them. 


\section{MODELING METHOD Synopsis}

The Modeling Method aims to correct many weaknesses of the traditional lecturedemonstration method, including the fragmentation of knowledge, student passivity, and the persistence of naive beliefs about the physical world.

\section{What to teach: Model-centered instructional objectives}

- To engage students in understanding the physical world by constructing and using scientific models to describe, to explain, to predict, to design and control physical phenomena.

- To provide students with basic conceptual tools for modeling physical objects and processes, especially mathematical, graphical and diagrammatic representations.

- To familiarize students with a small set of basic models as the content core of physics.

- To develop insight into the structure of scientific knowledge by examining how models fit into theories.

- To show how scientific knowledge is validated by engaging students in evaluating scientific models through comparison with empirical data.

- To develop skill in all aspects of modeling as the procedural core of scientific knowledge.

\section{How to teach: Student-centered instructional design}

- Instruction is organized into modeling cycles which engage students in all phases of model development, evaluation and application in concrete situations - thus promoting an integrated understanding of modeling processes and acquisition of coordinated modeling skills.

- The teacher sets the stage for student activities, typically with a demonstration and class discussion to establish common understanding of a question to be asked of nature. Then, in small groups, students collaborate in planning and conducting experiments to answer or clarify the question.

- Students are required to present and justify their conclusions in oral and/or written form, including a formulation of models for the phenomena in question and evaluation of the models by comparison with data.

- Technical terms and representational tools are introduced by the teacher as they are needed to sharpen models, facilitate modeling activities and improve the quality of discourse.

- The teacher is prepared with a definite agenda for student progress and guides student inquiry and discussion in that direction with "Socratic" questioning and remarks.

- The teacher is equipped with a taxonomy of typical student misconceptions to be addressed as students are induced to articulate, analyze and justify their personal beliefs.

BOX 2. 


\section{Teaching for Structured Knowledge: The Modeling Method}

Physics can be characterized as a complex network of models interrelated by a system of theoretical principles. Models are units of structured knowledge used to represent observable patterns in physical phenomena. Accordingly, "physical understanding" is a complex set of modeling skills, that is, cognitive skills for making and using models. The primary objective of physics teaching should therefore be to develop student modeling skills for making sense of their own physical experience and evaluating information reported by others. The Modeling Method of instruction (Box 2) has been developed to meet this objective.

To implement the instructional objectives in Box 1 effectively, teachers need to be equipped with clear ideas about models and modeling along with many examples of both. These matters are addressed in the next two sections. Teachers also need strategies and tactics for managing classroom activities. That is discussed in section 8 , but it takes Workshop experience to fully appreciate the skills involved.

Systematic implementation of the modeling method requires some restructuring of the physics curriculum. To make the structure and content of physics as explicit as possible, course material is organized around a small number of "basic models." For the mechanics portion of our high school physics course we use the five single particle models listed in Box 3. These are subdivided into five kinematical models to clarify the distinction between kinematics and dynamics as well as between descriptive and causal models. Instruction is designed to make students intimately familiar with the structure and use of the basic models. That includes using them to construct and analyze more complex models. It provides them with a set of clear examples grounded in personal experience from which they can develop general concepts of models and modeling in physics.

\section{Models}

The word "model" is used so frequently and expressively by physicists, especially on the research frontier, that it obviously refers to something important. However, some use the term so loosely as not to discriminate between models and

\begin{tabular}{ll}
\multicolumn{1}{c}{ Basic Particle Models in Newtonian Mechanics } \\
Kinematical Models & Causal Models \\
Constant velocity & Free Particle: $\Sigma \mathbf{F}_{i}=0$ \\
Constant acceleration & Constant force: $\Sigma \mathbf{F}_{i}=$ constant \\
Simple Harmonic Oscillator (SHO) & Linear binding force: $\Sigma \mathbf{F}_{i}=-k \mathbf{r}$ \\
Uniform circular motion (UCM) & Central force (with constant $|\mathbf{r}|$ ) \\
Collision $\Delta \mathbf{p}=\mathbf{I}$ & Impulsive force
\end{tabular}

BOX 3. 
theories (6). To capture the concept for the purpose of instruction, we provide it with a precise definition: A model (in physics) is a representation of structure in a physical system and/or its properties. The system may consist of one or more material objects or massless entities such as light. Unlike a theory, a model refers to an individual system, though that individual may be an exemplar for a whole class of similar things.

The concept of model is predicated on a philosophy of scientific realism holding that the universe is populated by things with definite physical properties. Physicists learn about things by investigating their properties empirically and framing their conclusions in terms of models and theories. They understand physical things through representations in the structure of validated models. Although scientific realism has been challenged by some philosophers, it is doubtful that physics research makes sense without it.

To complete our definition of model we still need to clarify the concepts of "structure" and "representation." Models in every domain of physics are characterized by the four types of structure listed in Box 4. Some commentary is needed to illuminate the structural types and the various representations used to specify them in a model. Examples are given in Box 5. It is especially important to note (in Box 5) how representation of structure is distributed across several different diagrammatic and mathematical representations. Thus, complete

\section{Model Specification}

A model is a representation of structure in a physical system and/or its properties. It describes (or specifies) four types of structure, each with internal and external components:

(a) systemic structure specifies

- composition (internal parts of the system)

- $\quad$ environment (external agents linked to the system)

- connections (external and internal causal links)

(b) geometric structure specifies

- $\quad$ position with respect to a reference frame (external geometry)

- $\quad$ configuration (geometric relations among the parts)

(c) temporal structure specifies change in state variables (system properties)

- $\quad$ descriptive models represent change by explicit functions of time

- $\quad$ causal models specify change by differential equations with interaction laws

(d) interaction structure specifies interaction laws expressing interactions among causal links, usually as function of state variables 
specification (and understanding) of a model requires coordination of multiple representations

\section{(a) Systemic Structure}

Every complete model includes a system schema specifying the composition, environment and connections of the system (Box 5). Though system schemas can be specified verbally, for systems of any complexity tabular and/or diagrammatic representations are more informative. Diagrammatic techniques for this purpose have evolved in all the sciences without recognition of their common cognitive function. For example, a standard electrical circuit diagram is a system schema. It specifies constituent parts of the system and electrical connectivity (causal links) among them. Similarly, chemists have developed elaborate representational tools for constructing molecular system schemas, and in biology, for example, animal digestive and circulatory systems can hardly be understood without diagrams.

Strangely enough, diagrams are seldom used to represent system schemas in elementary mechanics, perhaps because the systemic structure of a simple mechanical system is deemed to be so obvious to physicists. It is not so obvious to students, however. A common source for student failure to solve mechanics problems can be traced to their inability to identify agents of force on an object (5) — in other words, their inability to construct (in their heads) appropriate system schemas.

To construct a system schema from a given physical situation, or even from an artificially simple situation described in a "word problem," can be deceptively difficult; for it requires a judicious choice of system, identification of relevant properties and suppression of irrelevant information. It is actually a complex skill requiring extensive modeling to develop to a high level. Having students routinely construct and discuss diagrammatic system schemas like those in Box 5 may help them develop that skill. (This is a new idea that has not yet been tested in practice.) The schema in Box 5 specifies a system composed of two objects connected by a string $(\mathrm{S})$ and interacting with three external agents: table(T), earth (E) and pulley (P). Note that the closed dotted line serves to separate the system from its environment.

System schemas should not be confused with maps, which represent other kinds of information about systems. The schema in Box 5 may be regarded as implicit in the situation map (in the same Box), so it can be extracted (or abstracted) from the map, though this involves a choice in identifying the system of interest. When system structure is easily "read off" a map, a schema diagram may be superfluous. Nevertheless, its construction may serve as a check that something obvious has not been overlooked, or that everyone is talking about the same system.

\section{(b) Geometric Structure: Geometric Models}

Spatial location and configuration represent geometric properties possessed by all systems, though some models (for example, electrical circuit 


\section{Representations of structure in a model for the modified Atwood machine}

Situation Map

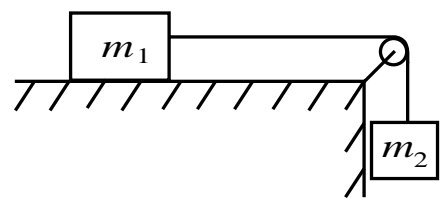

Motion Map

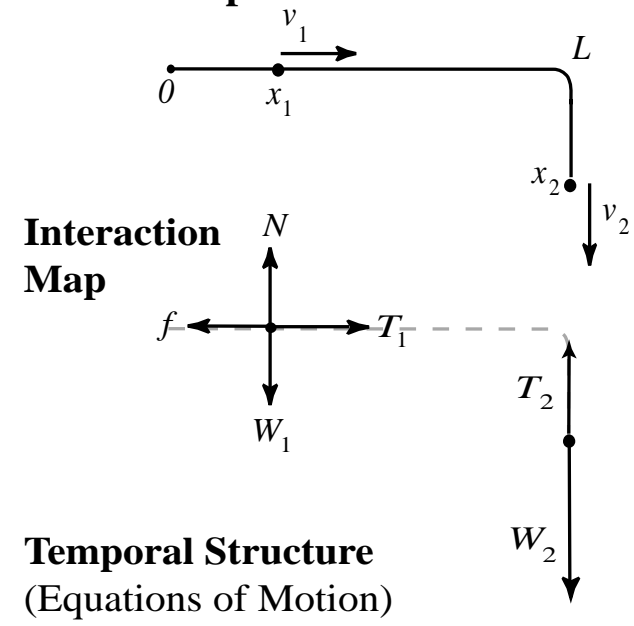

For single particle subsystems:

For entire 2 particle system:

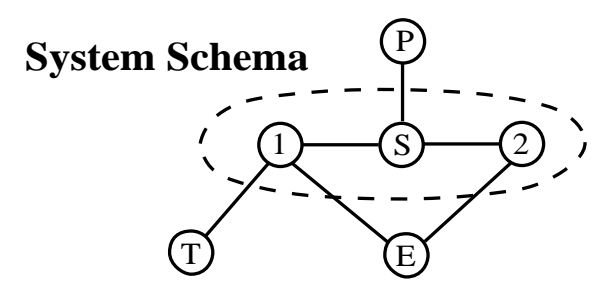

Subsystem Schema<smiles></smiles>

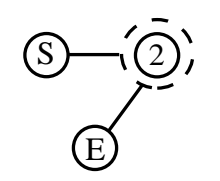

Geometric Structure

$$
\begin{aligned}
x_{2} & =x_{1}+L \\
\Rightarrow v_{2} & =v_{1} \\
\Rightarrow a_{2} & =a_{1}
\end{aligned}
$$

\section{Interaction Laws}

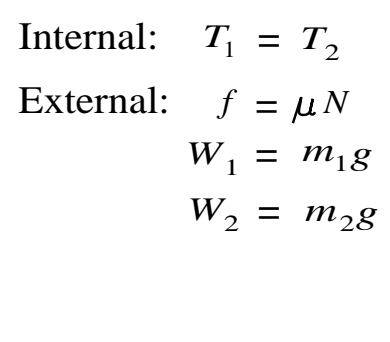

$$
\begin{aligned}
T_{1}-\mu N & =m_{1} a_{1} \\
m_{2} g-T_{2} & =m_{2} a_{2} \\
N & =m_{1} g
\end{aligned}
$$

$m_{2} g-\mu m_{1} g=\left(m_{1}+m_{2}\right) a_{1}$

\section{BOX 5. (see text for explanation)}

models) ignore geometric properties. On the other hand, purely geometric models (such as maps and architectural plans) ignore interactions and temporal structure.

Newtonian theory presumes that geometric properties of all objects can be modeled within a 3D Euclidean space. This has been called the Zeroth Law of physics (6). Relativity theory requires a profound modification of the Zeroth Law, but that will not concern us here. 


\section{Temporal Structure in the Constant Acceleration Model}

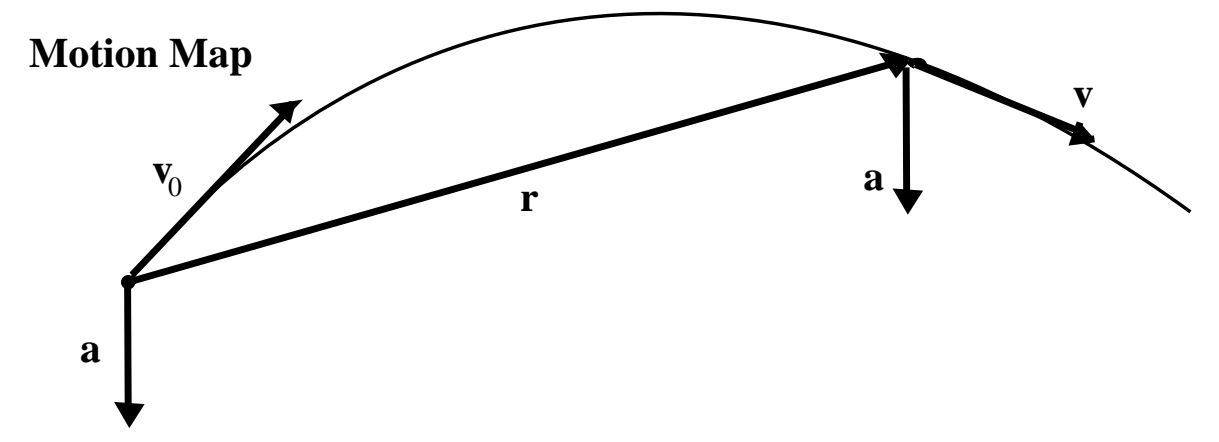

Parametric equation: $\mathbf{r}=\mathbf{v}_{0} t+\frac{1}{2} \mathbf{a} t^{2}$

Temporal structure: 2-time (2-point) relations

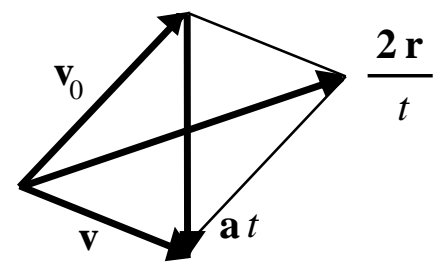

$$
\begin{aligned}
& \mathbf{v}+\mathbf{v}_{0}=\frac{2 \mathbf{r}}{t} \\
& \mathbf{v}-\mathbf{v}_{0}=\mathbf{a} t
\end{aligned}
$$

\section{BOX 6.}

Mathematical representations of geometric structure employ vectors or coordinate systems, but these are invariably coordinated with maps of diagrams to (i) designate an interpretation for the mathematical symbols, and (ii) help the user visualize the structure as a whole. Note that the geometric structure in Box 5 is distributed across several representations. Geometric structure relevant to the model has been extracted from the situation map and represented in a motion map, wherein the objects are represented as particles and their positions $\left(x_{1}, x_{2}\right)$ on the path of motion are designated. The positions are related to the length $L$ of the string. For an inextensible string (implicitly a model of interaction structure in the string) it follows immediately that the velocities and accelerations of the particles are equal.

\section{(c) Temporal Structure: Process Models}

State variables are descriptors representing basic properties that change with time. The state of a system at a particular time is described by the values of its state variables at that time. A model of temporal structure provides a comparison of states at different times. We can distinguish two kinds of model for temporal structure: descriptive and causal. 
$A$ descriptive model gives the state variables as explicit functions of time. For example, all the kinematic models listed in Box 3 are descriptive models. For the constant acceleration model, temporal structure of the state variables ( $\mathbf{x}$ and $\mathbf{v}$ ) is specified explicitly by vector-valued functions of time in Box 6. It is also given by a motion map, which depicts the values of kinematical quantities along the path of the particle.

A causal model specifies a change in state variables by equations of change, which determine the change as a consequence of interactions. Causal models are explanatory models, where the cause explains the change of state. Newton's Second Law $\mathbf{F}=m \mathbf{a}$ is the prototypical prescription for an equation of change, where the $\mathbf{F}$ represents cause and the a represents effect. It becomes a determinate equation of motion (change of place) when force laws are used to specify $\mathbf{F}$ as a function of the state variables ( $\mathbf{x}$ and $\mathbf{v})$.

A process model is a model for change in a single property (energy, for example) without reference to the underlying systems that possess that property. This can lead to error, as in the pseudoscience Energetics, which holds that energy is the primal stuff of which everything is made. Process models are therefore incomplete models, so to understand them students must know how to extend them to complete models. For example, in a process model of energy storage and transfer, students must be able to identify the carriers of the energy and the systems in which it is stored.

Motion (represented as change of position) is the most fundamental kind of change, because time itself is measured by standardized motion in an object called a clock. Relativity theory reduces temporal structure to geometric structure by fusing space and time into a single model of spacetime structure. However, that will not concern us here.

\section{(d) Interaction Structure: Interaction Models}

Interactions between systems are usually modeled by expressing the interaction variables as functions of state variables for the systems. Such "interaction laws" are needed to determine equations of change for the systems. In Newtonian mechanics, where the interaction variables are forces, the interactions are modeled by force laws, such as Hooke's law or Newton's law of gravitation. Alternatively, interactions can be modeled by potential energy functions. Other kinds of interaction involve the exchange of mass, charge or other physical quantities.

In Box 5 the interaction structure is distributed across two kinds of representation. First, the forces on each object are represented by force diagrams on an "interaction map." Second, magnitudes of the forces are specified by a set of interaction (force) laws. Note that the directions of the forces are specified by arrows on the map and not by any mathematical symbols or relations. This one more example of mixed representations employed in specifying a model. 


\section{Modeling and Modeling Tools}

Scientific understanding emerges from making and using models, in short, from modeling. To learn science, therefore, students must engage in all aspects of modeling. Modeling instruction is designed to promote that. To see what such a design involves, in this section we discuss three major modeling modes: model construction, analysis and validation. This has implications for problem solving as well as for the design of modeling tools and software to enable modeling.

Modeling in science is a complex process, requiring coordination of several kinds of modeling activities. Most, if not all, of the procedural knowledge implicit in scientific practice can be classified into some mode of modeling. Indeed, modeling constitutes the heart of the scientific method (6).

It has often been argued that instruction in the scientific method should be the primary purpose of science education, so students can learn to "think like a scientist." Unfortunately, implementation of this objective in science curricula has foundered on the lack of an adequate characterization of "scientific method." Indeed, as researchers in the history of science and cognitive science document the complexities of scientific practice, some have argued that there is no such thing as the scientific method - that there is not one method but many.

Modeling theory provides a new perspective on the methodology of science. A detailed analysis of modeling modes provides a rich characterization of the scientific method as a coherent system of procedural knowledge for constructing and using models in any scientific domain. The complexity of scientific practice is then seen to arise from specialization of some modeling tools to limited domains and the special skills required to use such tools.

A clear explication of the various modeling modes makes it possible to design instructional sequences to promote the acquisition of coordinated modeling skills. We address that issue in the next section.

\section{Model construction}

Modeling begins with a physical situation which we wish to understand. Understanding comes from making, analyzing and evaluating a model for the situation. The situation may be presented to us in any form; for example, as a lab experiment or a textbook problem. Whatever the situation, we may need to disregard some information as irrelevant or introduce additional information (e.g. relevant physical knowledge). From examining the situation, our first problem is to come up with (i.e. adduce) a suitable model in any way we can. Sometimes we can simply adapt a familiar model to the new situation; otherwise the problem is more difficult. In any case, our understanding of what constitutes a model (Box 4) is a sure guide for what to do.

On examining the situation the first thing to do is "Identify the system!" This is old advice known to every experienced science teacher, but it neglects to say what comes next. Box 5 tells us that we should next represent the system we have identified in a system schema. Then we will have specified precisely what it is that we intend to model.

Old advice tells us next to "Identify the variables!" Strictly speaking, this 


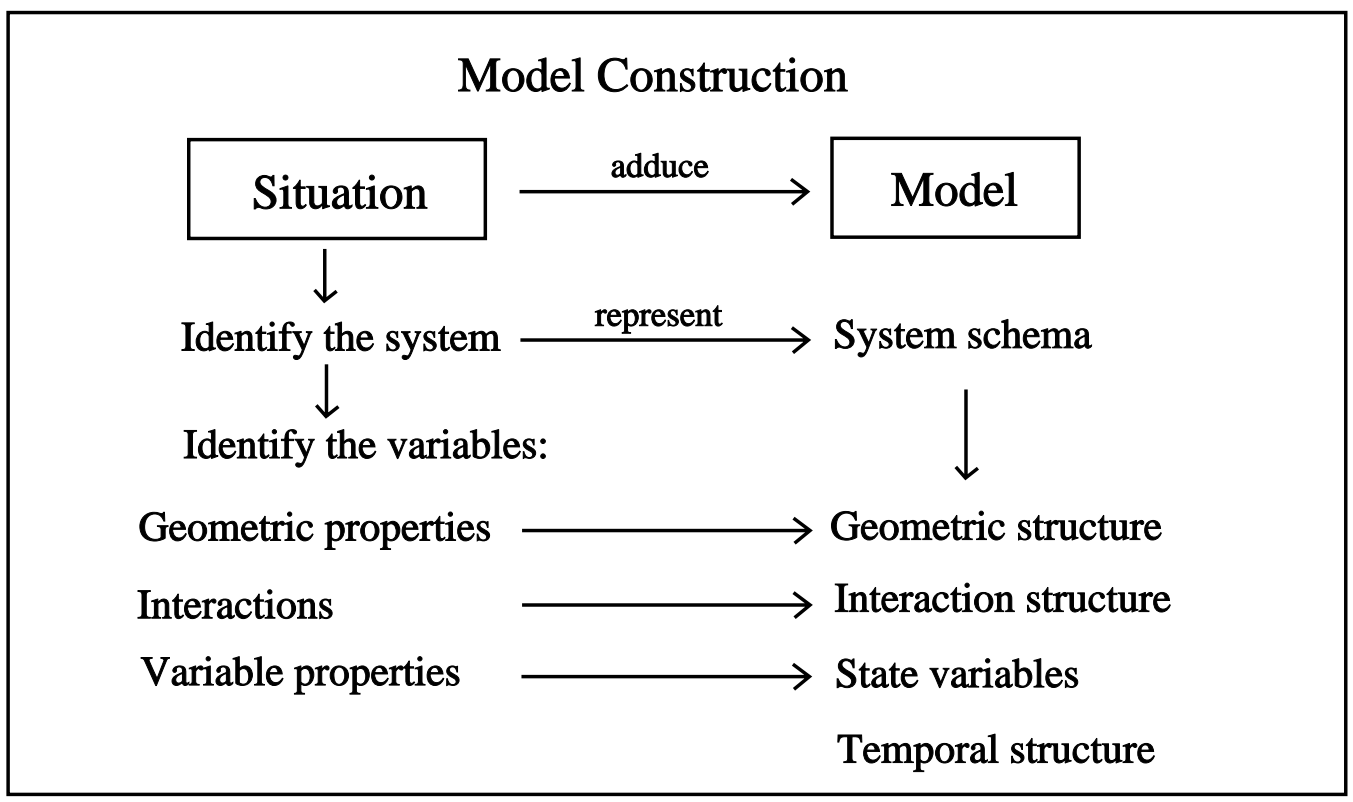

BOX 7.

advice should be phrased "Identify the properties of interest and specify variables to represent them." More specifically, as indicated in Box 7, the advice should be expanded to "Examine the geometry, interactions and variable properties of the system in order to represent their structure faithfully in the model." As explained below, the quality of the resulting model depends, in part, on the quality of the available modeling tools.

An example of the detailed model resulting from this model construction process is laid out in Box 5 for the situation presented there.

\section{Model analysis}

When a model has been constructed it must be analyzed to understand its structure and implications. The extraction of information or implications from a model is called model-based inference. All scientific predictions are inferences from models. An ordinary city map is a descriptive model, and using it to determine a route between two places is model-based inference. This can be regarded as a non-propositional form of deductive inference. The analysis of explanatory models can be very involved, even when the formulation of the model is quite simple. Some physicists devote their careers to model analysis.

For an example of model-based inference in mechanics, consider the constant acceleration model in Box 6, where the temporal structure is represented explicitly. Every question that can be asked of the model can be answered by inference from the two-time(two-point) parallelogram or the equivalent pair of vectorial equations. Details are given by Winans (17), so we can concentrate on general observations here. The representation in Box 6 should be compared with the coordinate-based representations in most textbooks. Not only is it more compact, but it is a better starting point for inferences. 
For example, a standard textbook problem is to calculate projectile range from given initial conditions. The textbooks derive a "range formula" which most students try to memorize instead of recognizing that the method of derivation is more important than the result. Indeed, the range formula applies only to horizontal displacements, and calculating the range to targets which are not on the horizontal is unnecessarily complicated by coordinate methods. Winans (17) shows how to get the general solution by graphical or trigonometric methods which are accessible to high school students. Essentially, it is the geometric method of "solving a triangle" for sides or angles, or rather, "solving a rectangle" (which is composed of several triangles). Actually, the most efficient way to do this is by using vector algebra, though that is not ordinarily appropriate for high school students, except in a second year "Advanced Placement" course. For example, from the vectorial equations for the parallelogram in Box 6 we can use the "inner product" to obtain $\left(\mathbf{v}+\mathbf{v}_{0}\right) \cdot\left(\mathbf{v}-\mathbf{v}_{0}\right)=(2 \mathbf{r} / t) \cdot(\mathbf{a} t)$, hence $\mathrm{v}^{2}-\mathrm{v}_{0}{ }^{2}=2 \mathbf{r} \cdot \mathbf{a}$. This formula relates sides of the parallelogram to the lengths and included angle of the diagonals. It illustrates the fact that the ease of inference depends on the representational and computational tools at one's disposal.

When vector multiplication is not available, the vectorial equations (in Box 6) must be decomposed into components in order to use elementary algebra to "solve them." Even so, the vectorial equations are useful because of their direct relation to the motion map and the two-time parallelogram, and these display the geometric structure of the model without irrelevant features of a coordinate system. Coordinate methods are overused in introductory physics. The only introductory textbook that consistently puts vectors ahead of coordinates is Alonso and Finn (1), but it is for calculus-based physics. A more advanced textbook by Hestenes (4) shows how to do all of classical mechanics without coordinates, but this requires new and more powerful rules for multiplying vectors.

\section{Model validation}

Validation is the process of assessing the adequacy of a model to represent a particular system and situation. It involves comparing the structure and predictions of the model to empirical data about the structure and behavior of the system it represents. If a match is confirmed to some level of accuracy the model is said to be validated to that degree. A perfect match is not to be expected, because every model is an incomplete representation, that is, there are always some features of the system which are not represented by the model. Moreover, the experimental apparatus for measuring a system's properties always has a limited accuracy. A full assessment of a model's validity will include an assessment of what is neglected in the model as well as the accuracy of the representation.

\section{Problem solving}

Traditional physics courses lay heavy emphasis on problem solving. This has the undesirable consequence of directing student attention to problems and 
their solutions as units of scientific knowledge. Modeling theory tells us that these are the wrong units; the correct units are models. Problem solving is important, but it should be subservient to modeling. Indeed, the various modeling modes raise a variety of problems, so problems can be classified according to their roles in modeling processes. It tells us that most physics problems are solved by constructing or selecting a model from which the answer to the problem is extracted by model-based inference. In a profound sense the model provides the solution to the problem. In fact, a single model solves many problems, and a handful of models suffice to solve most of the problems in introductory physics (e.g. Box 3). Thus, an emphasis on models and modeling simplifies the problem of organizing a physics course into understandable units.

Educational research has established that students' main difficulty in problem solving is in the preliminary "qualitative analysis" of a problem. We maintain that this qualitative analysis is best described as "setting up a model" from which the solution to the problem can be inferred. Besides providing a universal approach to problem solving, this view directs attention to the crucial role of representational tools in problem solving, or better, in modeling.

Reif (12) observes that even physics professors often fail to display good technique in solving elementary problems, and he recommends a prescriptive approach to teach optimal methods. We would modify his recommendations to prescribing a modeling approach to problem solving with emphasis on skill in using representational tools, to which we direct our attention next.

\section{Modeling tools}

Since models are representations of structure, we need modeling tools to construct the representations. Teachers are familiar with standard representational tools - verbal, mathematical, graphical and others. However they need to pay greater attention to the critical role of representational type and structure in developing physical understanding. It should be recognized that one's ability to model, and so to understand, depends on the tools at one's disposal. Therefore, teachers must be prepared to introduce new tools when students are ready to make good use of them. Instruction must be designed to develop student skills in wielding the tools.

We have seen that specialized modeling tools have been developed to represent different kinds of structure in models: system schema for system structure, motion maps for temporal structure, force diagrams for interaction structure. Motion maps and force diagrams have proved their value in years of modeling instruction and in the classrooms of other physics teachers. However, the instructor must take care to see that students learn to use these tools properly, for students, and, as we have seen in our Workshops, most teachers do not immediately see their value and use (5).

Among other modeling tools that have not been mentioned already, energy diagrams and bar charts are especially valuable for representing interaction structure, along with state transition diagrams for temporal structure. Graphs, of course are valuable for revealing structure in data by representing it in geometric 
form where it can be apprehended as geometric structure. In fact the very notion of structure is derived from geometry. However, graphs are sometimes used where other tools would do better. For example, motion maps are often more informative than kinematic graphs, though many textbooks do not exploit them systematically, and students frequently get the two confused.

It is obvious that progress in experimental science depends heavily on the development of more powerful instruments, from electron microscopes to radio telescopes. It is seldom noted, though, that progress in theoretical science is equally dependent on the development of more powerful modeling tools. It is not sufficient to call on mathematics to supply the tools. Each science needs new tools tailored to its own tasks. This is most evident in the development of specialized maps and diagrams peculiar to each science.

The heavy dependence of scientific practice on the available tools is not reflected in the typical science curriculum, which fails to discriminate not only between models and theories, but also between facts, models and the tools that produce them. No wonder that students come to view science as a motley collection of facts.

The modeling method aims to change that view by making models and modeling tools as explicit as possible in the curriculum, and by empowering students to understand and apply science by using these tools. The modern personal computer has immense potential to facilitate these objectives. It is a representational medium of unprecedented capacity and flexibility. Modeling theory can serve as a guide to exploiting this medium in the future, a guide for the design of computer modeling tools with unprecedented power. Some tools can be created easily by adapting existing software. Others will have to be designed expressly to facilitate particular modeling tasks. As major developments in this direction are to be expected in the near future, a brief overview may help us to exploit them.

From our discussion of modeling modes it is evident that computer modeling tools fall naturally into two classes:

(a) Tools for constructing and analyzing models. Of course, standard verbal, mathematical, graphical and diagrammatic tools have already been implemented in software. More significant are the possibilities for new representational modes that cannot be realized without computers. One that is already receiving a lot of attention is computer simulation. Another is the extension of graphs to $2 D$ and $3 D$ visualization of functional relations. Computers can make diagrams as easy to manipulate as mathematical symbols. They can also generate smart symbols and equations, which carry mathematical syntax along with them. These last two possibilities have seldom been noted, let alone exploited.

(b) Model validation tools are used to compare the predictions of given models to empirical data. These include data representation such as spread sheets, graphs and maps, which are universally recognized as superior to old manual representations. These general purpose tools have been further specialized into more powerful tools with already proven pedagogical value in physics instruction; 
for example, Microcomputer-Based Laboratory (MBL) tools that combine data acquisition with real time graphing. Such tools have already been incorporated into the modeling method.

\section{Planning and Managing Instruction}

The main objective of modeling instruction is to develop student skills in making and using models to understand the physical world. The fairly abstract analysis of structure in models and modeling activities in preceding sections is an essential prelude to the design of effective instruction. For we must know the structure of knowledge in order to teach it. The design of instruction, however, involves many other factors, especially an understanding about how students learn.

The modeling view is that students learn best from activities that engage them in actively constructing and using structured representations to make sense of their own experience and communicate with others. To optimize learning, the activities must be carefully planned and managed by the teacher. This requires considerable knowledge and skill on the part of the teacher which our Workshops are designed to cultivate. Here we can only sketch some of the main considerations. A list of instructional guidelines is given in Box 2.

The instructional modeling cycle (Box 2) is designed to teach scientific inquiry. It is similar to other teaching methods in presenting to students interesting physical phenomena to investigate collaboratively. It differs in the structuring and guiding of activities by the teacher. From the beginning of a cycle the teacher engages the student in giving an accurate description of the phenomena in question - identifying the relevant systems, components and variables. Students are guided to an investigation of structure in the phenomena, and, as needed, the teacher introduces appropriate tools for representing that structure. For example, one of the best ways to learn kinematics is by using MBL tools to investigate motion. These tools produce real time graphical representations, which facilitate immediate comparison of the graphs with the motion. The teacher's job is to help students "see" such representations as models of the motion and understand how they can be converted to reproducible (best fit) mathematical models. It is well established that, without suitable teacher guidance, the technological benefits of MBL are lost on the students (Hake, 3). Likewise, teacher guidance is essential throughout the modeling cycle. Gradually, over several cycles, the students are led to see that the outcome of every investigation is a model which must be clearly delineated and evaluated. They gradually internalize the steps in conducting an investigation (procedural knowledge about science), so they become progressively more independent of the teacher. Soon they are ready for deeper insights into physics, as comes from using Newton's laws to model just about anything.

A typical modeling cycle is at least two weeks long with about a week for each phase. The mechanics course is organized into a sequence of modeling cycles built around the models listed in Box 3. There are two phases in the 
modeling cycle: development and deployment. The development phase is devoted to constructing and evaluating a model for some physical system. Typically it involves the design and execution of an experiment followed by an oral presentation and critique of results. The deployment phase involves applying the model in a variety of new situations to explain, predict, design or control. Problem solving is incorporated in this phase, often with class discussion and analysis.

The instructional modeling cycle is not intended to duplicate cycles of model building and testing in research, but it does incorporate the various modes of modeling in a systematic way that reflects the procedural structure of scientific inquiry. The modeling cycle is thus a plan for organizing instruction into structurally coherent units. It is a flexible plan that can be adapted to various instructional objectives. For example, It is not necessary to begin every cycle with an experimental investigation. Alternatively, the cycle could begin with the presentation of a model to be analyzed, or with a design problem. The beginning, however, is always a situation to be attacked with modeling skills and tools.

A detailed example of a modeling cycle is described in Wells et. al. (16). That cycle began with an investigation of the modified Atwood machine. As described there, the students do not get involved in modeling the Atwood machine with the sophistication and detail of Box 5. However, sometime after that cycle when the students have been introduced to the whole set of Newton's laws, and have expanded their repertoire of modeling tools, it would be a good idea to revisit the Atwood machine with an analysis of the model structure as laid out in Box 5. This illustrates how to deepen student understanding by using new conceptual tools for a richer analysis of previous experience.

The most critical element in successful implementation of the modeling method is the skill of teacher in managing classroom discourse. The modeling method aims to promote skill in scientific discourse by engaging the students repeatedly in discussions and presentations about modeling real systems. The expert teacher moderates student discourse carefully to insure that its quality, as judged by scientific standards, continues to improve. The first standard governs the quality of descriptions. Students learn to replace vague descriptive terms of ordinary language with precisely defined scientific terms like "system, interaction, velocity and force." And to articulate reasons for doing so. They learn to coordinate these terms with increasing skill in generating coherent scientific descriptions, including the specification of system schema and descriptive variables. Further, standards of scientific discourse govern the quality of inference, explanation and argumentation. Such standards are implicit in discourse within the scientific community, but they are seldom articulated explicitly, and there is no consensus as to what they are.

A primary objective of scientific discourse is to formulate and evaluate scientific claims. This requires (i) models for precise formulation of claims, (ii) methods for analyzing the claims, and (iii) data for evaluating the claims. It is a process of constructing arguments and evaluating evidence to produce justified 
belief. Developing student skills in this process may be the most important objective of science instruction.

The first step in promoting classroom discourse is to establish a climate of openness and respect in which every student feels encouraged to participate. Special measures may be required to engage reluctant or reticent students. Personal opinions must be solicited so that they can be examined and evaluated objectively and publicly. Participation is enhanced when students have a personal stake in the course and outcome of the discourse. Strong feedback from peers and teacher induces students to clarify, defend or modify their beliefs.

Effective communication requires shared meaning. Section 2 reviewed conclusive evidence that students and teacher do not attach the same meanings to technical terms like "force" at the beginning of a physics course and often at the end. Meanings (of words, equations, diagrams) are constructed from situated use by each student individually. Therefore, to establish a common understanding of important terms and representations their meanings must be negotiated in open discourse. This is essential to give every student access to the intellectual content of the course.

There are many modes of discourse, including whole class and small group discussions, reviews and summaries, prepared presentations and critiques. The teacher decides what mode is most conducive to the objectives of the day. The teacher establishes the subject of discourse, guides it toward the objective and seeks to bring it to a satisfying closure.

The objective of discourse may be to formulate or analyze some "target model" or representation. Discourse is most effective when it is centered on some artifact and/or melded with some activity. This is called situated discourse. An excellent example is given by Roth (14). He challenged his class to a tug of war using a block and tackle (giving him the mechanical advantage, of course). The students became greatly excited and animated. He easily induced them to sketch and debate a flurry of diagrams to explain his advantage until they reached a consensus on the explanation. This consensus could not have been achieved without the public representation of structural relations in the diagrams. This is a good example of how the quality of discourse and the structure of arguments depend on the representational tools. Scientific argumentation arises naturally when the students have the modeling resources. This must be engineered by the teacher.

\section{Conclusions}

The National Research Council has recently published National Standards for K-12 science course content and for the professional development of teachers (11). The program described in this paper is fully in accord with those standards and exceeds them in some respects.

The main message of this paper is that:

- Informed teacher planning and guidance is the key to effective instruction. (A good physics course cannot be "canned" in a textbook or curriculum materials.) 
- To learn the structure of physics, students must be continually engaged in making and using representations of structure (models) for specific physical systems and processes. Public presentation, examination and critique of the models is the key to consolidating understanding.

The course described in this paper has emerged from a program of physics education research and active collaboration with high school teachers. Such collaboration is essential for effective implementation of research findings and a powerful incentive for new research. Consequently, the course is under continual development. Indeed, some of the specifics described in this paper have not yet been implemented, and more is in the works.

Acknowledgment: I am indebted to my collaborators on the Modeling Workshop Project — Larry Dukerich, Ibrahim Halloun, Jane Jackson and Gregg Swackhamer — for stimulating and contributing many ideas in this paper, and especially for making the project a success. This work was supported by a grant from the National Science Foundation.

\section{References}

(1) Alonso, M. and Finn, E., Physics, Reading Addison-Wesley, 1992.

(2) Clement, J. \& Camp, C., Preconceptions in Mechanics, Dubuque, Kendall/Hunt, 1994.

(3) Hake, R., Interactive-engagement vs traditional methods: A six-thousand-student survey of mechanics test data for introductory physics courses. Am. J. Phys. (submitted).

(4) Hestenes, D., New Foundations for Classical Mechanics, Dordrecht/Boston, Kluwer, 1986, 3rd printing with corrections, 1992 (to be republished by STAR Publ., Cambridge, 1996).

(5) Hestenes, D., Toward a Modeling Theory of Physics Instruction. Am. J. Phys. 55: 440-454, 1987.

(6) Hestenes, D., Modeling Games in the Newtonian World, Am. J. Phys. 60: 732-748, 1992.

(7) Hestenes, D., and Jackson, J., These Proceedings (1996).

(8) Hestenes, D., Wells, M. \& Swackhamer, G., Force Concept Inventory, The Physics Teacher 30: $141-158,1992$.

(9) Johnson, M., The body in the mind, Chicago, U. Chicago Press, 1987.

(10) Lakoff and Johnson, Metaphors we live by, Chicago, U. Chicago Press, 1980.

(11) National Research Council, National Science Education Standards, Wash. DC, National Academy Press, 1996.

(12) Reif, F. , Understanding and teaching important scientific thought processes. Am. J. Phys. 63: 17-32, 1995.

(13) Reif, F. , Standards and measurements in physics - Why not in physics education?

Am. J. Phys. 64: 687-688, 1996.

(14) Roth, W-M. , Drawing Diagrams, Waving Hands \& Talking Pulleys: Learning to Theorize Simple Machines. Interactive Learning Environments (to be published, 1996).

(15) Simon, H. \& Larkin, J., Why a Diagram is (Sometimes) Worth Ten Thousand Words. Cognitive Science 11: 65-99, 1987.

(16)Wells, M., Hestenes, D. \& Swackhamer, G., A modeling method for high school physics instruction, Am. J. Phys. 63: 606-619, 1995.

(17)Winans, J., Trajectories without trigonometry, The Physics Teacher (Sept.) 341-343, 1971. 